\title{
MODELO INTEGRADO PARA LOCALIZAÇÃO HIERÁRQUICA DE HUBS EM REDES DE FLUXOS
}

\author{
Guina Sotomayor Alzamora \\ Instituto Nacional de Matemática Pura e Aplicada (IMPA) \\ Estrada Dona Castorina 110 - Jardim Botânico - RJ, Brasil \\ guinas@gmail.com \\ Fernanda Raupp \\ Laboratório Nacional de Computação Científica (LNCC) \\ Avenida Getúlio Vargas 333 - Quitandinha, Petrópolis - RJ, Brasil \\ fernanda@lncc.br
}

\begin{abstract}
RESUMO.
O problema de localização de hubs visa encontrar pontos de concentração de fluxos numa rede, de modo que a soma das ligações compartilhadas entre os pontos da rede seja minimizada. Neste trabalho, um modelo matemático para o problema fundamental de localização hierárquica de hubs com alocação simples numa rede de fluxos é apresentado. O modelo proposto integra as decisões sobre localização de hubs regionais e centrais, visando a minimização do número de conexões de fluxo na rede. Experimentos computacionais com o modelo proposto são realizados para instâncias dos sistemas de transporte aéreo americano e brasileiro, usando o solver GLPK e um software com recursos gráficos de implementação própria. Resultados numéricos preliminares indicam o potencial do modelo integrado aqui proposto.
\end{abstract}

Palavras-chaves: problema de localização de hubs, localização hierárquica

Área principal: L\&T - Logística e Transportes

\begin{abstract}
.
The hub location problem aims to find points of concentration of a network flow so that the sum of the connections shared between the network points is minimized. In this work, a mathematical model for the fundamental problem of hierarchical locating hubs with simple allocation in a network flow is presented. The proposed model integrates the decisions on locating regional and central hubs, aiming at minimizing the total links flow in the network. Computational experiments with the proposed model are conducted for instances of the American and the Brazilian air transportation systems, using the GLPK solver and a own software implemented with graphical features. Preliminary numerical results show the potential of the integrated model proposed here.
\end{abstract}

Keywords: hub location problem, hierarchy location

Main area:L \& T - Logistics and Transport 


\section{Introdução}

Estamos interessados em estudar o problema de localização de hubs com alocação simples em redes de fluxo. Hubs ou concentradores são introduzidos para reduzir o número de conexões entre nós origem e destino [13], quando comparados a uma rede sem hubs. De fato, a introdução de hubs em uma rede permite consolidar fluxos, gerando um número menor de conexões, e consequentemente aumentando a eficiência da rede, uma vez que permite decrementar significativamente custos operacionais com a economia de escala gerada [4]. Ainda, pode-se pensar em introduzir concentradores que interagem numa configuração de mais de uma camada [10], permitindo a hierarquização de funcionalidades.

Uma visão geral da pesquisa sendo desenvolvida sobre problemas de localização de hubs pode ser encontrada em [11], [12] e [7]. Neste último, se faz um overview dos últimos 25 anos de pesquisa de localização de $h u b s$, introduzido formalmente por O'Kelly [21], e fornece reflexões sobre suas origens, comentários sobre o presente, e sugere direções promissoras para os próximos 25 anos. Outros trabalhos de revisão da literatura interessantes são [20], [1], que categorizaram os problemas de localização de hubs em quatro grandes subgrupos: problema $q$-hub mediana, problema de localização de hubs com custos fixos, problema $q$-hub centro e problema de hubs de cobertura, [17] e [6], nos quais pode-se verificar que muitos modelos sofisticados têm sido propostos. Farahani et al. [13] apresentam uma revisão e classificação de modelos, técnicas de solução, assim como aplicações de problemas de localização de hubs e futuras tendências. Ainda, Şahin e Süral oferecem uma revisão sobre sistemas de localização hierárquica [10].

Neste trabalho, o problema fundamental de localização hierárquica de hubs com alocação simples em redes de fluxos é abordado, via apresentação de um modelo que integra as decisões de localização de hubs centrais e regionais. Vale lembrar que no problema fundamental de localização de hubs, proposto originalmente por Campbell [5], não se considera fluxos diretos, mas sim fluxos que passam por pelo menos um e no máximo dois hubs. Quando existem duas categorias de hubs, os fluxos devem passar por pelo menos um hub regional e no máximo por dois hubs centrais, no caso de alocação simples, ou seja, cada ponto da rede é conectado a exatamente um único hub.

Aqui, redes de fluxos são sistemas de transporte aéreo, e fluxos são passageiros transportados. Na tentativa de superar o desafio para melhor atender a demanda crescente por esses sistemas, e consequentemente melhorar suas eficiências, propõe-se a estratégia de localização hierárquica de hubs. Com a introdução de hubs centrais e regionais nesses sistemas, espera-se que os aeroportos com baixo fluxo de passageiros sejam alocados a aeroportos com movimento maior, os hubs regionais, que por sua vez são alocados a aeroportos com fluxo ainda maior, os hubs centrais, consolidando fluxos de passageiros de forma eficiente.

A demanda por transporte aéreo no mundo superou a marca de 2.738 milhões de passageiros em 2011 e, desde 2002, o volume mundial anual de passageiros tem aumento em média $5,1 \%$ [18]. No caso brasileiro, a demanda anual por transporte aéreo apresentou uma média de crescimento igual a 10,6\%, muito superior à média mundial, entre os anos $2002 \mathrm{e}$ 2011. Sem investimentos em infraestrutura que acompanhassem tal crescimento, verificouse, nos últimos anos, o surgimento de gargalos operacionais e limitações de capacidade nos principais aeroportos brasileiros, comprometendo os fluxos de carga e de passageiros no país [25].

Neste cenário, muitas companhias aéreas possuem dificuldades em planejar e programar voos que atendam satisfatoriamente seus clientes, uma vez que há aeroportos com amplo volume de tráfego somente em horas pico, e existem outros que atingem sua capacidade de uso durante muitas horas do dia [15]. Para que essas restrições de capacidade nos aeroportos não se tornem uma barreira para o atendimento da demanda crescente 
por serviços do sistema de transporte aéreo no futuro, é razoável considerar a estratégia de inserção de hubs pelas companhias aéreas individualmente ou pelo responsável pela gerência de todo o sistema de transporte aéreo.

Ainda, experimentos computacionais com o modelo proposto são realizados, tendo como instâncias o sistema de transporte aéreo americano [21] e o brasileiro [3], levando em conta o conhecimento das localizações geográficas (latitude e longitude) dos aeroportos, das distâncias entre os aeroportos, dos fluxos de passageiros e das quantidades de hubs regionais e centrais a serem localizados. A escolha do número de aeroportos candidatos a serem hubs regionais e centrais é feita baseada no fluxo mínimo de passageiros, de acordo com [3] e [9]. O modelo integrado proposto foi resolvido com o software GLPSOL, versão 4.8, do pacote GLPK. O método de solução aplicado foi o algoritmo de branch-andcut, método exato para resolver problemas de programação inteira mista. Os resultados obtidos são visualizados num software desenvolvido com recursos gráficos.

O artigo está organizado da seguinte forma. Na Seção 2, o problema de localização de hubs é apresentado juntamente com seus principais modelos. O modelo proposto que integra as decisões de localizar hubs centrais e regionais é apresentado na Seção 3. Os experimentos numéricos realizados com o modelo integrado são apresentados na Seção 4. Finalmente, na Seção 5, são apresentados os comentários finais deste trabalho.

\section{Modelos de localização}

Na área da Pesquisa Operacional, os problemas de localização de facilidades são bastante abordados, sob o ponto de vista de desenvolvimento de modelos, de métodos de solução e aplicações.

Conhecido geralmente por problema de localização de hubs, o problema de desenho de rede de fluxos do tipo hub-and-spoke trata da localização de hubs e da alocação de nós da rede (spokes) aos hubs, de modo a satisfazer a demanda e otimizar um ou mais critérios [4]. As alocações podem ser do tipo simples ou múltipla; no caso de simples, os nós spokes são alocados a exatamente um único hub, enquanto que no caso de múltipla, os nós spokes podem ser alocados a mais de um hub.

Segundo Campbell e O'Kelly [7], as principais características dos problemas de localização de hubs são: (i) Demandas estão associadas a fluxos entre pares origem-destino, e não de forma pontual. (ii) Os fluxos são autorizados a passar por instalações de hubs. (iii) Hubs são instalações a serem localizadas na rede. (iv) Existe um benefício no roteamento de fluxos através de hubs. (v) O objetivo depende da localização de hubs e do roteamento de fluxos. (vi) Fluxos entre pares origem-destino passam por um ou dois hubs. (vii) Fluxos diretos entre pares origem-destino não são permitidos. Ao adicionar estas duas últimas características, Campbell [5] definiu o "problema fundamental de localização de hubs", o qual é aqui estudado.

Antes de apresentar o modelo integrado para a localização hierárquica de hubs numa rede de fluxos, vamos destacar a seguir os modelos que serviram de base para o desenvolvimento do modelo a ser proposto.

\subsection{O modelo da $p$-mediana}

O modelo matemático mais popular associado ao problema de localização é o modelo da p-mediana e suas primeiras formulações foram apresentadas por Hakimi [16] e Kariv e Hakimi [19]. Em Pizzolato et al. [22], uma revisão sobre as aplicações dos problemas de localização que utilizam o modelo da p-mediana, variantes desse modelo e métodos de solução é apresentada; em Reese [23] são apresentados métodos de solução e uma diversificada gama de aplicações suplementares desse modelo. 
Na versão simplificada do modelo, fixa-se o número de instalações ou medianas $p$ a serem localizadas no conjunto de pontos dados, de modo a minimizar a soma ponderada das distâncias dos clientes, centrados em pontos do conjunto, às instalações que prestarão serviços.

Sejam os parâmetros: $N$ o conjunto de $n$ pontos ou nós dados, e seus índices $i, j \in N ; p(<n)$ o número de medianas a serem localizadas; $w_{i}$ o peso associado a cada ponto $i$; e $\left[d_{i j}\right]_{n \times n}$ a matriz simétrica das distâncias, em que cada elemento $d_{i j}$ é a distância do ponto $i$ ao ponto $j$, com $d_{i i}=0, \forall i$. Considere ainda a ser determinada a matriz de alocações $\left[z_{i j}\right]_{n \times n}$, onde $z_{i j}=1$, se o ponto $i$ é alocado a $j$, e $z_{i j}=0$, caso contrário; $z_{j j}=1$ indica que $j$ é uma mediana e $z_{j j}=0$ indica o caso contrário. Então, o modelo da $p$-mediana pode ser formulado como segue:

$$
\begin{array}{lll}
\operatorname{minimizar} & \sum_{i \in N} \sum_{j \in N} w_{i} d_{i j} z_{i j} & \\
\text { sujeito a } & \sum_{j \in N} z_{i j}=1 \quad i \in N \\
& \sum_{j \in N} z_{j j}=p & \\
& z_{i j}-z_{j j} \leq 0 \quad i, j \in N \\
& z_{i j} \in\{0,1\} \quad i, j \in N,
\end{array}
$$

onde o objetivo (1) é minimizar a soma ponderada das distâncias entre os pontos dados e os pontos selecionados como medianas; as restrições em (2) indicam que cada ponto $i$ é conectado somente a uma única mediana $j$; a restrição (3) garante que há exatamente $p$ medianas; as restrições em (4) afirmam que cada ponto deve ser atribuído a uma mediana, e, finalmente, variáveis de decisão binárias são impostas em (5).

Para Contreras e Fernandez [8] a modelagem de problemas de localização de hubs tem por base o modelo clássico da $p$-mediana.

\subsection{O modelo $q$-hub-mediana}

Usando como justificativa que o modelo de relaxação linear proposto por Campbell [5] resultava em soluções muito fracionadas para o problema fundamental de localização de hubs, Skorin-Kapov et al. [24] propuseram um modelo de programação inteira mista para resolver o problema fundamental de localização de hubs baseado no modelo da $p$ mediana, conhecido como modelo $q$-hub-mediana. Neste modelo são introduzidos dois tipos de variáveis: inteiras para modelar decisões de localização e contínuas para modelar fluxos na rede.

Sejam os parâmetros: $N$ o conjunto de $n$ nós ou pontos dados, e seus índices $i, j, k, m \in N ; W_{i j}$ o fluxo entre os nós $i$ e $j ;\left[d_{i j}\right]_{n \times n}$ a matriz simétrica das distâncias, em que cada elemento $d_{i j}$ é a distância do nó $i$ ao nó $j ; \alpha$ o fator de desconto para o fluxo entre hubs; e $q$ o número de hubs a serem localizados. Considere ainda as variáveis de decisão $y_{i j k m}$ como sendo a fração do fluxo de $i$ a $j$ que passa pelos hubs $k$ e $m$ nesta ordem, se $k=m$, então o fluxo passa por um único $h u b$; e $x_{i k}=1$ como sendo o nó spoke $i$ alocado ao $h u b k$, e $x_{i k}=0$, no caso contrário; ainda $x_{k k}=1$ indica que $k$ é um $h u b$, e $x_{k k}=0$ o caso contrário. Então, o modelo de $q$ - $h u b$ mediana é formulado por: 


$$
\begin{gathered}
\operatorname{minimizar} \sum_{i \in N} \sum_{j \in N} \sum_{k \in N} \sum_{m \in N} W_{i j}\left(d_{i k}+d_{j m}+\alpha d_{k m}\right) y_{i j k m} \\
\sum_{k \in N} x_{k k}=q \\
\sum_{k \in N} x_{i k}=1 \\
\sum_{m \in N} y_{i j k m}=x_{i k} \\
\sum_{k \in N} y_{i j k m}=x_{j m} \\
x_{i j} \leq x_{j j} \\
y_{i j k m} \geq 0 \\
x_{i k} \in\{0,1\}
\end{gathered}
$$

onde a função objetivo (6) a ser minimizada representa os custos relativos aos fluxos entre cada par origem-destino. Por (7) um número fixo igual a $q$ de hubs deve ser localizado. Em (8) as restrições asseguram que cada nó é alocado a exatamente um único hub. As restrições (9) asseguram que $x_{i k}$ é factível se o nó $i$ é alocado ao $h u b k$ independentemente do destino, enquanto que as restrições em (10) asseguram que o fluxo é factível somente se $j$ é alocado ao hub $m$ independentemente da origem. Em (11) as restrições garantem que um nó somente é alocado a um hub já localizado. As restrições em (12) impõem frações de fluxo não negativas, enquanto que as restrições em (13) definem variáveis de alocação de tipo binário.

Observe que o modelo (6)-(13) corresponde a um problema de programação inteira mista. Porém, Skorin-Kapov et al. [24] relatam que, em experimentos computacionais realizados com o modelo acima, os valores ótimos de $y_{i j k m}$ são majoritariamente inteiros, e que quando apresentavam valores fracionados, os valores ótimos da função objetivo variavam em menos de $1 \%$ abaixo dos valores ótimos correspondentes.

\section{Modelo de localização hierárquica}

Nesta seção, o problema fundamental de localização hierárquica de hubs numa rede de fluxos é abordado, via a introdução de um modelo matemático que integra a localização simultânea de hubs regionais, para consolidação de fluxos menores, tendo por base o modelo da $p$-mediana, e de hubs centrais, para consolidação de fluxos maiores, tendo por base o modelo da $q$-hub mediana, visando a minimização do fluxo total percorrido.

Sejam os parâmetros: $N$ o conjunto de $n$ nós ou pontos dados, e seus índices $i, j, u, k, m \in N$; $p$ o número de hubs regionais a serem localizados; $q$ o número de hubs centrais a serem localizados; $W_{i j}$ o fluxo entre os nós $i$ e $j$; e $\left[d_{i j}\right]_{n \times n}$ a matriz simétrica das distâncias, em que cada elemento $d_{i j}$ é a distância do nó $i$ ao nó $j$. Considere ainda as variáveis de decisão: $z_{i u k}$ que indica a alocação do nó spoke $i$ ao hub regional $u$ conectado ao $h u b$ central $k ; x_{i u k}$ que representa a quantidade de fluxo com origem ou destino no nó $i$, que passa através do $h u b$ regional $u$ conectado ao $h u b$ central $k$; e $y_{i j k m}$ que representa a fração do fluxo do nó origem $i$ ao nó destino $j$ que passa pelos hubs centrais $k$ e $m$ nesta ordem, se $k=m$ então o fluxo passa por um único hub central. O modelo proposto é formulado como: 


$$
\begin{gathered}
\sum_{i \in N} \sum_{j \in N}\left(W_{i j}+W_{j i}\right) \sum_{u \in N} d_{i u} \sum_{k \in N} z_{i u k} \\
+\sum_{i \in N} \sum_{u \in N} \sum_{k \in N} \alpha_{R} d_{u k} x_{i u k}+\sum_{i \in N} \sum_{j \in N} \sum_{k \in N} \sum_{m \in N} \alpha_{C} W_{i j} d_{k m} y_{i j k m}
\end{gathered}
$$

sujeito a

$$
\begin{array}{cl}
\sum_{u \in N} \sum_{k \in N} z_{u u k}=p & \\
\sum_{u \in N} \sum_{k \in N} z_{i u k}=1 & i \in N \\
z_{i u k} \leq z_{u u k} & i, u, k \in N \\
\sum_{u \in N} z_{i u k} \leq z_{k k k} & i, k \in N \\
\sum_{k \in N} z_{k k k}=q & \\
\sum_{j \in N}\left(W_{i j}+W_{j i}\right)\left(z_{i u k}-z_{j u k}\right) \leq x_{i u k} & i, u, k \in N \\
\sum_{m \in N} y_{i j k m}=\sum_{u \in N} z_{i u k} & i, j, k \in N \\
\sum_{k \in N} y_{i j k m}=\sum_{u \in N} z_{j u m} & i, j, m \in N \\
z_{i u k} \in\{0,1\} & i, u, k \in N \\
x_{i u k} \geq 0 & i, u, k \in N \\
y_{i j k m} \geq 0 & i, j, k, m \in N,
\end{array}
$$

onde $\alpha_{R}$ e $\alpha_{C}$ são interpretados como fatores de economia de escala conseguida pela agregação de fluxos com a introdução de hubs regionais e centrais, respectivamente. A função objetivo (14) a ser minimizada representa o fluxo total percorrido entre spokes e $h u b s$ regionais, entre hubs regionais e hubs centrais e entre hubs centrais. A restrição (15) garante que há exatamente $p$ hubs regionais a serem localizados, já as restrições em (16) indicam que cada nó spoke $i$ é alocado a exatamente um único $h u b$ regional $u$ que por sua vez é alocado a um único hub central $k$. As restrições em (17) garantem que um nó spoke $i$ é alocado a um hub regional $u$ já localizado. As restrições em (18) garantem que um nó $i$ (spoke ou $h u b$ regional) seja alocado a um $h u b$ central $k$ já localizado. A restrição (19) garante que há exatamente $q$ hubs centrais a serem localizados. As restrições em (20) dizem respeito ao fluxo entre os hubs regionais e centrais para cada spoke, representado por $x_{i u k}$. As restrições em (21) asseguram que um hub central $k$ é factível, se um nó $i$ é alocado a ele independentemente do destino; enquanto que por (22) assegura-se que um $h u b$ central $m$ é factível, se um nó $j$ é alocado a ele independentemente da origem. As restrições (23) definem $z_{i u k}$ como variáveis de decisão de tipo binário, enquanto que as restrições $(24)$ e (25) indicam que as variáveis de decisões $x_{i u k}$ e $y_{i j k m}$ são não negativas.

Observe que, por este modelo, hubs centrais também são hubs regionais.

\section{Experimentos computacionais}

Nesta seção, são apresentados os experimentos computacionais com o modelo proposto para o problema de localização hierárquica de hubs em sistemas de transporte aéreo. Para isso, foram consideradas duas instâncias: 
- Instância CAB10 (ou simplesmente CAB), encontrada em O'Kelly [21]. Reúne 10 aeroportos do sistema de transporte aéreo dos Estados Unidos. Nesse sistema, são informados os nomes das cidades, e não os dos aeroportos, as coordenadas geográficas (latitude e longitude) de cada aeroporto, e os fluxos de passageiros existentes entre os aeroportos. Nesta instância, a matriz de fluxos é simétrica, ou seja, a quantidade de passageiros transportados é igual entre cada par de aeroportos $\left(W_{i j}=W_{j i}\right)$, existindo um total de 45 conexões distintas entre os aeroportos. A Tabela 1 apresenta as coordenadas geográficas dos aeroportos, e a Figura 1 mostra os fluxos diretos entre os aeroportos dessa instância. Cabe informar que a cor vermelha possui tonalidade mais clara em fluxos de passageiros menores e tonalidade mais escura em fluxos maiores. Vale observar que quanto mais largo for o semento, maior é o fluxo de passageiros.

Tabela 1: Coordenadas geográficas dos aeroportos de CAB10

\begin{tabular}{cccc}
\hline \hline Num. & Cidade & Latitude & Longitude \\
\hline \hline 1 & Atlanta & 33,7426 & $-84,3564$ \\
\hline 2 & Baltimore & 39,2949 & $-76,6121$ \\
\hline 3 & Boston & 42,3747 & $-71,0521$ \\
\hline 4 & Chicago & 41,8971 & $-87,6297$ \\
\hline 5 & Cincinnati & 39,0991 & $-84,5124$ \\
\hline 6 & Cleveland & 41,5003 & $-81,6951$ \\
\hline 7 & Dallas-Fort W. & 32,7549 & $-97,3280$ \\
\hline 8 & Denver & 39,7388 & $-104,9855$ \\
\hline 9 & Detroit & 42,3321 & $-83,04586$ \\
\hline 10 & Houston & 29,7572 & $-95,3711$ \\
\hline \hline
\end{tabular}

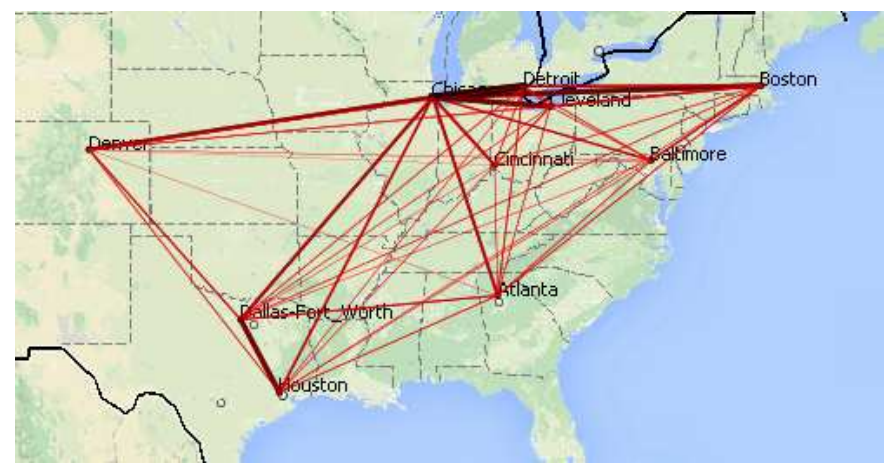

Figura 1: Fluxos diretos de passageiros em CAB10

- Instância BRA10, montada com informações do sistema de transporte aéreo brasileiro, é encontrada em Alzamora [2]. Neste sistema com 10 aeroportos, considera-se o código utilizado pela ICAO [18], e não o nome, para cada aeroporto, e são conhecidas as coordenadas geográficas (latitude e longitude), juntamente com os fluxos de passageiros existentes entre os aeroportos. Nesta instância, a matriz de fluxos não é simétrica, ou seja, a quantidade de passageiros transportados entre dois aeroportos pode ser diferente dependendo do sentido $\left(W_{i j} \neq W_{j i}\right)$, existindo um total de 44 conexões entre aeroportos, e não 45, isto porque uma das conexões tem fluxo nulo. A Tabela 2 apresenta as coordenadas geográficas dos aeroportos, enquanto que a Figura 2 mostra os fluxos diretos entre eles. Novamente, a tonalidade mais clara da 
cor vermelha indica fluxos menores e a tonalidade mais escura indica fluxos maiores. Vale observar que quanto mais largo for o segmento de reta, maior é o fluxo de passageiros.

Tabela 2: Coordenadas geográficas dos aeroportos de BRA10

\begin{tabular}{ccccc}
\hline \hline Num. & Aeroporto & Cidade & Latitude & Longitude \\
\hline \hline 1 & SBEG & Manaus, AM & $-3,0411$ & $-60,0506$ \\
\hline 2 & SBSV & Salvador, BA & $-12,9086$ & $-38,3225$ \\
\hline 3 & SBFZ & Fortaleza, CE & $-3,7758$ & $-38,5322$ \\
\hline 4 & SBBR & Brasília, DF & $-15,8692$ & $-47,9208$ \\
\hline 5 & SBBE & Belém, PA & $-1,3847$ & $-48,4789$ \\
\hline 6 & SBRF & Recife, PE & $-8,1264$ & $-34,9228$ \\
\hline 7 & SBCT & Curitiba, PR & $-25,5358$ & $-49,1714$ \\
\hline 8 & SBGL & Rio de Janeiro, RJ & $-22,81$ & $-43,2506$ \\
\hline 9 & SBPA & Porto Alegre, RS & $-29,9939$ & $-51,1711$ \\
\hline 10 & SBGR & Guarulhos, SP & $-23,4356$ & $-46,4731$ \\
\hline \hline
\end{tabular}

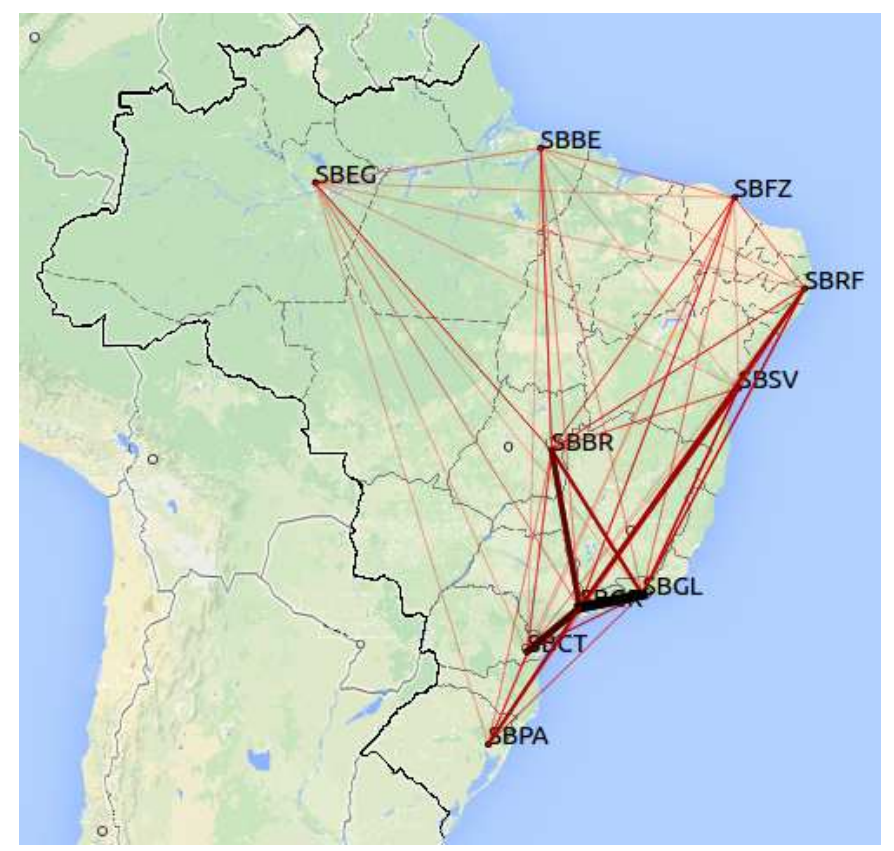

Figura 2: Fluxos diretos de passageiros em BRA10

O problema de localização hierárquica, cujo modelo integrado foi introduzido em (14)-(25), para as instâncias descritas acima, foi resolvido com o software GLPSOL, versão 4.8, do pacote GLPK. O método de solução aplicado foi o algoritmo de branchand-cut, método exato para resolver problemas de programação inteira mista. O sistema de visualização gráfica foi implementado na linguagem $\mathrm{C}++$, usando a ferramenta Qt para a interface gráfica. Além disso, os experimentos numéricos foram efetuados num computador Intel Pentium (R) Dual CPU E2140 @1.60GHz × 2 e 3.2 GiB de memória sob o sistema operacional de 64 bits, Linux (Ubuntu 14.04LTS).

Para os testes computacionais foram considerados: $p=5$ (número de hubs regionais a serem localizados), de acordo com o critério de volume mínimo de fluxo de passageiros de Alzamora et al. [3]; q variando de 2 a 4 (número de hubs centrais), baseado no estudo de Costa et al. [9]; $\alpha_{R}=1,0$ e $\alpha_{C}=0,6$ (fatores de economia de escala), 
semelhante a Figueiredo et al. [14]. Para ambas as instâncias testadas, os modelos gerados com $N=10$ aeroportos apresentaram 4.113 restrições, 12.000 variáveis, sendo 1.000 binárias.

Para CAB10 com $p=5$, os resultados de localização de hubs regionais para $q=2$ são: Chicago, Dallas-Fort W., Cincinnati, Detroit e Houston; para $q=3$ são: Chicago, Dallas-Fort W., Cincinnati, Detroit e Denver; e para $q=4$ são: Chicago, Dallas-Fort W., Boston, Cleveland e Denver. Já os resultados de localização dos hub centrais mostraramse consistentes: para $q=2$ foram eleitos Chicago e Dallas-Fort W., para $q=3$ foi acrescentado à lista anterior Denver, e para $q=4$ foi acrescido à lista Cleveland.

Na Figura 3 são apresentados os fluxos consolidados em apenas 10 conexões, contra 45 conexões iniciais, após a localização hierárquica de hubs referente à instância CAB10, com 10 aeroportos, 5 hubs regionais e 3 hubs centrais. Os segmentos de reta azuis mostram as conexões dos aeroportos spokes aos hubs regionais, os segmentos de reta verdes mostram os aeroportos que são hubs regionais alocados aos hubs centrais, e os segmentos de reta em lilás mostram as interconexões entre os hubs centrais. Vale observar que quanto mais largo for o segmento de reta, maior é o fluxo de passageiros.

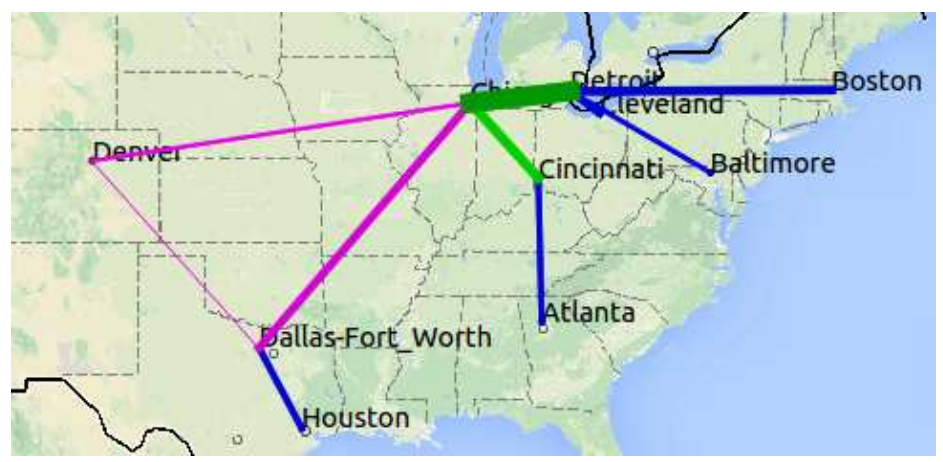

Figura 3: Fluxos consolidados com 5 hubs regionais e 3 hubs centrais para CAB10

Ainda, na Tabela 3, são informados os tempos de execução em segundos e os custos ótimos, ou seja, os valores ótimos da função objetivo (14) para a instância CAB10.

Tabela 3: Tempos de execução e custos ótimos para CAB10

\begin{tabular}{cccc}
\hline \hline p-hubs & $q$-hubs & Tempo (s) & Função Objetivo \\
\hline \hline 5 & 2 & 4,7 & $700.777 .358,5738$ \\
\cline { 2 - 4 } & 3 & 9,2 & $632.419 .707,0173$ \\
\cline { 2 - 4 } & 4 & 8,2 & $577.268 .389,1886$ \\
\hline
\end{tabular}

Para BRA10 com $p=5$, os resultados de localização de $h u b$ regionais foram idênticos para $q=3$ e $q=4$, sendo eles: SBSV, SBBR, SBCT, SBGL, SBGR. Os resultados de localização de $h u b$ regionais para $q=2$ diferem do anterior com relação a localização um hub regional, ao invés de SBGL foi eleito SBFZ. Já os resultados para localização de $h u b$ centrais mostraram-se consistentes: para $q=2$ foram eleitos SBSV e SBGR, para $q=3$ acrescentou-se SBBR à lista anterior, e para $q=4$ acrescentou-se SBGL à lista.

Na Figura 4 são apresentadas apenas 10 conexões de fluxo, contra 44 conexões iniciais, após a localização hierárquica de hubs referente à instância BRA10, com 10 aeroportos, 5 hubs regionais e 2 hubs centrais. Novamente, os segmentos de reta azuis mostram as conexões dos aeroportos spokes aos hubs regionais, os segmentos de reta verdes mostram os aeroportos que são hubs regionais alocados aos hubs centrais, e os segmentos 
de reta em lilás mostram as interconexões entre os hubs centrais. Vale observar que quanto mais largo for o segmento de reta, maior é o fluxo de passageiros.

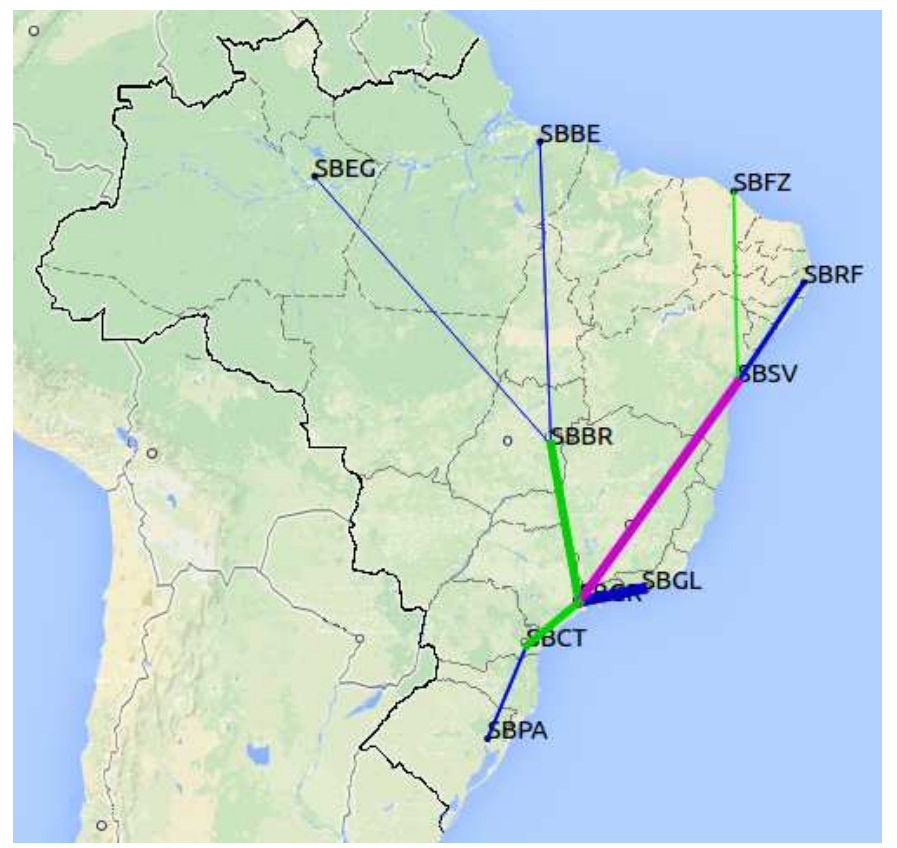

Figura 4: Fluxos consolidados com 5 hubs regionais e 2 hubs centrais para BRA10

Finalmente, na Tabela 4, são informados os tempos de execução em segundos e os percursos ótimos, ou seja, os valores ótimos da função objetivo (14) para a instância BRA10.

Tabela 4: Tempos de execução e percursos ótimos para BRA10

\begin{tabular}{cccc}
\hline \hline p-hubs & $q$-hubs & Tempo (s) & Função Objetivo \\
\hline \hline 5 & 2 & 3,7 & $70.114 .926 .622,652$ \\
\cline { 2 - 4 } & 3 & 2,4 & $61.149 .866 .128,423$ \\
\cline { 2 - 4 } & 4 & 2,0 & $55.733 .423 .160,381$ \\
\hline \hline
\end{tabular}

\section{Conclusões}

Neste trabalho, propõe-se uma nova estratégia para resolver o problema fundamental de localização hierárquica de hubs numa rede de fluxos com alocação simples, via a introdução e resolução de um modelo integrado. Também foi desenvolvido um software gráfico para visualizar os dados e resultados do problema abordado. Os resultados numéricos com duas instâncias de sistemas de transporte aéreo mostraram que o modelo integrado é promissor em otimizar conexões de fluxo de passageiros mediante a introdução de hubs regionais e centrais; as ligações iniciais foram bastante reduzidas.

Podemos verificar um certo desiquilíbrio na localização de $h u b$ centrais e regionais e na distribuição dos fluxos para a instância CAB10 quando comparada a instância BRA10. Acreditamos que isso se deva ao fato de que os fluxos entre os aeroportos obedecem a propriedade da desigualdade triangular, imposta no modelo através das restrições em $(20)$.

Os resultados apresentados verificam a importância da introdução de hubs em redes de fluxos, já que permite concentrar fluxos em rotas mais frequentes, reduzindo 
seus percursos. Pode se pensar que, com a passagem obrigatória por pelo menos um hub, alguns percursos fiquem maiores, porém isso é compensado com a consolidação de todos os fluxos, existindo ganho em escala com a redução de conexões.

Assim, não só hubs são introduzidos como também se faz uma categorização dos mesmos em hubs regionais, associados a fluxos menos densos, e em hubs centrais, associados a fluxos mais densos, com a tarefa de resolver um problema complexo, quando a rede de fluxo sendo considerada é de média e grande escala, permitindo hierarquização das operações aeroportuárias.

Como trabalhos futuros pretende-se introduzir a alocação múltipla, assim como novos critérios para a localização de hubs.

\section{Referências}

[1] Sibel Alumur and Bahar Y Kara. Network hub location problems: The state of the art. European Journal of Operational Research, 190(1):1-21, 2008.

[2] Guina Guadalupe Sotomayor Alzamora. Estratégias de localização de hubs para o sistema de transporte aéreo brasileiro. D. sc. thesis, PUC-Rio, RJ, 2013.

[3] Guina Sotomayor Alzamora, Fernanda M P Raupp, and Nelio D Pizzolato. Estratégia de localização de hubs no transporte aéreo brasileiro. In Simpósio Brasileiro de Pesquisa Operacional (SBPO), Natal, RN, 2013.

[4] Yu An, Yu Zhang, and Bo Zeng. The reliable hub-and-spoke design problem: Models and algorithms. Optimization Online, 2011.

[5] James F Campbell. Integer programming formulations of discrete hub location problems. European Journal of Operational Research, 72:387-405, 1994.

[6] James F Campbell, A Ernst, and M Krishnamoorthy. Hub Location Problems, chapter 12, pages 373-407. Springer, 2009.

[7] James F Campbell and Morton E O'Kelly. Twenty-five years of hub location research. Transportation Science, 46:153-169, 2012.

[8] Ivan Contreras and Elena Fernandez. General network design: A unified view of combined location and network design problems. European Journal of Operational Research, 219:680697, 2011.

[9] Tiago F G Costa, Gui Lohmann, and Alessandro V M Oliveira. A model to identify airport hubs and their importance to tourism in Brazil. Research in Transportation Economics, pages $3-11,2010$.

[10] Güvenç Şahin and Haldun Süral. A review of hierarchical facility location models. Computers 83 Operations Research, 34(8):2310-2331, 2007.

[11] Zvi Drezner, editor. Facility Location: A Survey of Applications and Methods. Springer Series in Operations Research. Springer Verlag, New York, 1995.

[12] Zvi Drezner and Horst W Hamacher, editors. Facility Location: Applications and Theory. Springer, New York, 2004.

[13] Reza Zanjirani Farahani, Masoud Hekmatfar, Alireza Boloori Arabani, and Ehsan Nikbakhsh. Hub location problems: A review of models, classification, solution techniques and applications. Computers \& Industrial Engineering, 64:1096-1109, 2013.

[14] Raphael M A Figueiredo, M E O'Kelly, and Nelio D Pizzolato. A two stage hub location model for air transportation on Brazil. International Transactions in Operational Research (ITOR), 21:275-289, 2014. 
[15] Marc C Gelhausen, Peter Bersten, and Dieter Wilken. Do airport capacity constraints have a serious impact on the future development of air traffic? Journal of Air Transport Management, 28:3-13, 2013.

[16] S L Hakimi. Optimum locations of switching centers and the absolute centers and medians of a graph. Operations Research, 12:450-459, 1964.

[17] Masoud Hekmatfar and Mirsaman Pishvaee. Hub Location Problem, chapter 11, pages 243-270. Physica-Verlag Heidelberg, 2009.

[18] ICAO. Annual report of the council 2011, 2012. Doc 9975.

[19] O Kariv and S L Hakimi. An algorithmic approach to network location problems. II: The p-medians. SIAM Journal of Applied Mathematics, 37:539-560, 1979.

[20] John G Klincewicz. Hub location in backbone / tributary network design: A review. Location Science, 6:307-335, 1998.

[21] Morton E O'Kelly. A quadratic integer program for the location of interacting hub facilities. European Journal of Operational Research, 32(3):393-404, 1987.

[22] Nelio Domingues Pizzolato, Fernanda M P Raupp, and Guina Sotomayor Alzamora. Revisão de desafios aplicados em localização com base em modelos da $p$-mediana e suas variantes. Pesquisa Operacional para o Desenvolvimento(PODES), 4(1):13-42, 2012.

[23] J Reese. Solution methods for the p-median problem: An annotated bibliography. Networks, 48(3):125-142, 2006.

[24] Darko Skorin-Kapov, Jadranka Skorin-Kapov, and Morton O'Kelly. Tight linear programming relaxations of uncapacitated $p$-hub median problems. European Journal of Operational Research, 94:582-593, 1996.

[25] Peter F Wanke. Efficiency of Brazil's airports: Evidences from bootstrapped DEA and FDH estimates. Journal of Air Transport Management, 23:47-53, 2012. 